\title{
Epidemiologia delle batteriemie nosocomiali in Lombardia nel triennio |999-200|
}

\author{
Egidio Franco Vigano' , Daniela Fossati, Massimo Colciago, Milena Arghittu, Marinella \\ Cainarca, Antonio Grossi, Francesco Luzzaro, Manuela Montuori, Angelo Sala, Panajota \\ Troupioti (Gruppo di studio delle Infezioni Nosocomiali dell'AMCLI Lombardia, \\ coordinatore E. F.Vigano' )
}

\author{
Gruppo di Studio delle Infezioni Ospedaliere dell'AMCLI Lombardia \\ Hanno partecipato allo studio: \\ D.Fossati, M. Colciago: Servizio di Patologia Clinica, Presidio di Carate-Giussano, A.O. Ospedale Civile di Vimercate (MI) \\ C.Sturla,M.Montuori Sezione di Microbiologia, Ospedale di Gallarate (VA) \\ A.Grossi, S.Bergamaschi, V.Biancini, M.Gippiali, R.Meriggi , Sezione di Microbiologia, Ospedale di Treviglio-Caravaggio (BG ) \\ E.F.Vigano', L.Bertinotti , U.O. Microbiologia, Ospedale di Legnano ( MI) \\ F.Luzzaro, G.Lombardi, A.Endimiani , U.O. Microbiologia, Ospedale di Circolo di Varese \\ P.Troupioti, Sezione di Microbiologia, Presidio di Sondalo, A.O. Valtellina e Valchiavenna (SO) \\ M.Arghittu, E.Bianchi, P. Cambieri Sezione di Microbiologia, Presidio Predabissi, A.O. Melegnano (MI) \\ A.Sala, C.Pianetta U.O. Microbiologia, Ospedale Manzoni di Lecco \\ M.Cainarca Sezione di Microbiologia, Ospedale S.Paolo di Milano
}

Key words bloodstreams infections, Bacteraemia, Epidemiology, Nosocomial infections

\section{SUMMARY}

We analyzed bloodstream infection(BI) performed in eight hospitals in Region Lombardia in the period 1999$200 \mathrm{I}$ following criteria and methods as in the study performed in the year 1997.Data were evaluated from 434.000 hospital patients (one third of hospital patients annualy recovered in Lombardia Region each year).

Of these 56.3 positive blood coltures / 1000 patients were observed. Data were similar as 1997. In total 3063 episodes of bacteriemia were observed $(6.98 \mathrm{BI} / 1000$ hospital patients).53\% of cases correlated to hospital infection.

Organism identification of causative organisms resulted $22.5 \%$ for E.coli and $10.6 \%$ for KES; $17.7 \%$ S.aureus,4.5\% Yeast .Anaerobic bacteria resulted involved in 3.4\%.Among the frequency of isolates difference was noted in different wards In intensive care unit E.coli was pathogenic in $5.9 \%$ of patients, but KES in $25.5 \%$ of cases.

Differences were noted if nosocomial or comunity infections were involved. It was concluded that epidemiological data of Bl from all the hospital of Region Lombardia could support the knowledge of bacteriemic episodes in different wards as useful method of surveillance particularly in order to control economic aspects and efficacy of prevention.

\section{INTRODUZIONE}

La batteriemia nosocomiale rappresenta una importante causa di morbilità e di mortalità.

È stato calcolato che la batteriemia costituisce almeno il $10 \%$ di tutte le infezioni nosocomiali (1) e che negli USA se ne osservano almeno 250.000 casi all'anno (2).

La batteriemia nosocomiale nei pazienti in terapia intensiva è associata ad una mortalità attribuibile del $35 \%$, ad un prolungamento della degenza di 24 giorni e ad un eccesso di costi ospedalieri di 40.000 dollari per sopravvissuto (3).

In generale i tassi di batteriemia variano in relazione al tipo di ospedale ed alla tipologia dei pazienti e sono stimati tra 4 e 8 casi per 1.000 ricoveri (4).

Uno studio di prevalenza condotto in Europa da Bouza ( 5 ) nel 1998 su 9590 ricoveri aveva evidenziato un tasso di batteriemia molto superiore
(27,2 casi per 1.000 ricoveri) con il $72,8 \%$ di questi episodi classificati come nosocomiali : va detto tuttavia che gli ospedali partecipanti allo studio erano prevalentemente universitari e che più del $20 \%$ dei pazienti ricoverati erano stati sottoposti ad emocolture .

Uno studio di incidenza della sepsi condotto in Italia su 20.601 pazienti nel 1997 in 41 ospedali (6) aveva dimostrato 136 casi di batteriemia, pari a 6,6 casi per 1.000 ricoveri, di cui il $46 \%$ nosocomiali.

Uno studio di prevalenza delle infezioni nosocomiali, condotto in Lombardia nel 2000 dalla Direzione Generale Sanità (7), su 18.682 ricoveri aveva evidenziato un tasso di infezione del sangue (definizione clinica) nosocomiale del 5,8 per 1.000 con un tasso di batteriemia del 3 per 1.000 . Nelle terapie intensive i tassi di infezione del sangue nosocomiale erano del 70 per 1.000 . 
Poiché la identificazione di caso di batteriemia richiede l'isolamento di un microrganismo dal sangue attraverso l'emocoltura, poiché l'emocoltura rappresenta uno dei più importanti esami effettuati nei laboratori di Microbiologia, sia come impegno tecnico che come costo, riteniamo che da una analisi epidemiologica dei dati di laboratorio si possano ricavare informazioni utili sia per i clinici (terapia empirica) che per chi deve occuparsi di politica sanitaria (prevenzione delle batteriemie e riduzione dei costi).

Nel 1997 il Gruppo di studio sulle Infezioni Ospedaliere dell'AMCLI ha condotto uno studio epidemiologico in 9 Ospedali della Lombardia su 144.501 ricoveri, evidenziando 6,33 casi di batteriemia per 1.000 ricoveri (8); lo stesso gruppo ha poi studiato le resistenze antibiotiche osservate nel 1999-2000 in 2.796 batteri isolati da emocolture (9).

Scopo del lavoro attuale del Gruppo dell' AMCLI è la descrizione della epidemiologia delle batteriemie in un triennio in Lombardia, utilizzando la stessa metodologia del 1997 su un campione molto più numeroso, per analizzare l'andamento delle batteriemie nosocomiali e fare proiezioni in termini di frequenza e costi delle stesse nella nostra Regione.

\section{MATERIALI E METODI}

Ospedali: hanno partecipato allo studio l'Ospedale Civile di Lecco, l'Ospedale di Circolo di Legnano, l'Ospedale S. Antonio Abate di Gallarate, l'Ospedale Predabissi di Melegnano, l'Ospedale S.Paolo di Milano, L'Ente Ospedaliero Bormio e Sondalo, 1'Ospedale TreviglioCaravaggio, l'Ospedale di Circolo di Varese.

Sono stati inclusi nello studio tutti i pazienti con un ricovero $>1$ giorno nel periodo 1999 - 2001

Definizione di caso: sono state utilizzate le stesse metodologie del 1997 (8) che ribadiamo:

episodio infettivo : momento in cui si effettuano le emocolture ; tutte le emocolture effettuate nello stesso paziente in 7 giorni vengono considerate un unico episodio infettivo;

prelievo : set di bottiglie (o altri dispositivi) utilizzati per una singola venipuntura;

batteriemia significativa: isolamento di un battere o fungo sicuro patogeno (es. S.aureus) anche da un solo prelievo o di possibili patogeni o contaminanti tipo Stafilococchi coagulasi negativi o Streptococchi alfa emolitici o Corynebatteri se isolati da due o più prelievi per episodio infettivo; pseudobatteriemia: isolamento di un possibile patogeno o contaminante da un solo prelievo (sull'unico o su due o tre effettuati);

batteriemia polimicrobica: isolamento nello stesso episodio infettivo di 2 o più microrganismi con caratteristiche da batteriemia significativa batte- riemia nosocomiale: batteriemia da prelievi effettuati dopo $>72$ ore dal ricovero in ospedale.

Prelievi per emocolture : non sono stati utilizzati protocolli comuni di prelievo ed ogni ospedale ha continuato a lavorare nei tre anni dello studio utilizzando proprie metodologie per la emocoltura e le successive identificazioni batteriche.

\section{RISULTATI}

\section{Epidemiologia}

Sono stati studiati complessivamente 434.466 ricoveri di $>1$ giorno, in cui sono stati definiti 24.280 episodi infettivi, pari a 56,35 episodi per 1.000 ricoveri.

Il numero medio di prelievi di emocolture è stato di 2,1 per episodio, per un totale di 51.408 prelievi per emocoltura nei tre anni.

Complessivamente abbiamo osservato 3.033 casi di batteriemia significativa, pari a 6,98 batteriemie per 1.000 ricoveri. Il $53 \%$ delle batteriemie erano nosocomiali, pari a 3,71 casi per 1.000 ricoveri. Il 5,6 \% delle batteriemie erano polimicrobiche. Infine abbiamo definito come pseudobatteriemie ben 1.470 casi, pari al $6 \%$ degli episodi infettivi. Nella tabella 1 sono riportati i dati, confrontati con quelli del 1997. I dati tra parentesi rappresentano il range delle osservazioni nei vari ospedali. Nella tabella 2 osserviamo l'epidemiologia del triennio 1999-2001 per raggruppamenti di reparti. Le medicine contribuiscono al $38 \%$ dei ricoveri ma in questo raggruppamento troviamo il $61 \%$ degli episodi infettivi ed il $60 \%$ delle batteriemie significative, prevalentemente comunitarie $(57 \%)$.

Nei reparti chirurgici solo 16 pazienti su 1.000 vengono sottoposti ad emocolture e tuttavia la \% di emocolture positive (batteriemie / episodi infettivi) è maggiore $(17,1 \%)$ rispetto ai reparti medici, per il fatto che probabilmente il chirurgo ricorre all'emocoltura solo in presenza di forti sospetti di sepsi e per il fatto che gli episodi sono per 2/3 nosocomiali (437 su 631 totali).

Nelle terapie intensive 274 pazienti su 1.000 vengono sottoposti ad emocolture, le positività sono elevate come nelle chirurgie $(17,2 \%)$ e le sepsi sono nosocomiali nel $83 \%$ dei casi.

Nelle pediatrie infine il ricorso alla emocoltura è pari alle medicine (96 episodi per 1.000 ricoveri) tuttavia le positività sono minori $(5,38 \%)$ e prevalentemente comunitarie $(62,8 \%)$.

Eziologia

Nella tabella 3 osserviamo che E.coli è il $1^{\circ}$ in frequenza $(22,5 \%)$, seguito da S.aureus $(17,7 \%)$ e $\operatorname{KES}(10,6 \%)$. Di rilievo appare il fatto che il 4,5\% delle setticemie sono dovute a lieviti e che gli anaerobi sono responsabili del 3,4\% delle batteriemie.

Se analizziamo l'eziologia separando le infezioni nosocomiali dalle comunitarie osserviamo spostamenti significativi. E.coli è responsabile del $33 \%$ 
delle comunitarie e solo del $13 \%$ delle nosocomiali, S.pneumoniae è il $3^{\circ}$ in frequenza nelle comunitarie $(9,3 \%)$ ma solo il $12^{\circ}$ nelle nosocomiali $(1,1$ $\%)$. S.aureus nettamente primo nelle batteriemie nosocomiali, seguito da KES e Stafilococchi coagulasi negativi, con E.coli solo $4^{\circ}$.

Nella ultima colonna della tabella 3 è esposto il rapporto in frequenza tra batteriemie nosocomiali e comunitarie per i vari microrganismi, che consente di apprezzare ancora più chiaramente il diverso ruolo eziologico: $i$ lieviti sono 7 volte più frequenti nelle batteriemie nosocomiali rispetto alle comunitarie, gli Stafilococchi coagulasi negativi 3,8 volte, Ps.aeruginosa 3,34 volte e il gruppo KES 2,94 volte.

All'opposto le batteriemie da Salmonella sono solo comunitarie, quelle da S.pneumoniae lo sono quasi sempre (rapporto 0,13 ) e quelle da E.coli lo sono prevalentemente (rapporto 0,45 ).

Nella tabella 4 possiamo osservare le differenze in eziologia nei 4 raggruppamenti di reparto considerati: E.coli è $1^{\circ}$ in frequenza in Medicine, Chirurgie e Pediatrie ed è solo $6^{\circ}$ nelle Terapie Intensive; S.aureus è $2^{\circ}$ in tutti i raggruppamenti, a conferma della sua rilevanza eziologica in tutte le categorie di pazienti. Significativa appare nelle Chirurgie la elevata frequenza dei lieviti ( $10,1 \%)$ rispetto agli altri raggruppamenti, così come quella degli anaerobi $(6,1 \%)$.

Nelle Terapie Intensive il gruppo KES è addirittura il $1^{\circ}$ in frequenza $(25,5 \%)$ e sono elevate le frequenze di Stafilococchi coagulasi negativi $(18,4$ \%) e Ps.aeruginosa (10,5\%). Nelle Pediatrie infine da sottolineare la elevata frequenza di S.pneumoniae $(14,9 \%)$ e di Salmonella $(8,2 \%)$. Nella tabella 5 la eziologia nei diversi raggruppamenti è scomposta in Nosocomiali e Comunitarie: questa presentazione consente di apprezzare le differenze rispetto al dato complessivo della tabella 4.

Ad esempio nelle Medicine E.coli ha un complessivo del 26,4 \% ma passa dal $15,8 \%$ delle Nosocomiali al $35 \%$ delle Comunitarie, S.aureus (8,5 \%) passa dal $24 \%$ delle Nosocomiali al $14 \%$ delle Comunitarie. Nelle Terapie Intensive il gruppo KES , $1^{\circ}$ in frequenza con $25,5 \%$ sul totale, passa a 30,0\% delle Nosocomiali e solo 5,3\% delle Comunitarie.

Lascio le altre osservazioni alla attenta lettura della tabella.

Tabella I. Epidemiologia

\begin{tabular}{|c|c|c|}
\hline & $\begin{array}{c}\text { Studio AMCLI Lombardia } \\
\text { anni } 1999 \text { - 200I }\end{array}$ & $\begin{array}{c}\text { Studio AMCLI Lombardia } \\
\text { anno } 1997\end{array}$ \\
\hline$\overline{\mathrm{N}^{\circ} \text { ricoveri }>\text { I giorno }}$ & 434.466 & $|44.50|$ \\
\hline $\mathrm{N}^{\circ}$ episodi infettivi & 24.480 & 7.600 \\
\hline Episodi infettivi per 1.000 ricoveri & $56,35(40,4-83,8)$ & $52,6(39-69,3)$ \\
\hline N.prelievi emocolture per episodio & $2,1(1,60-3)$ & $2, I(I, 7-2,4)$ \\
\hline $\mathrm{N}^{\circ}$ batteriemie significative & 3.033 & 916 \\
\hline \% batteriemie /episodi infettivi & $12,39 \%(9,17-22,2)$ & $12,1 \%(8,5-15,9)$ \\
\hline $\mathrm{N}^{\circ}$ pseudobatteriemie & 1470 & 570 \\
\hline \% pseudobatteriemie / episodi infettivi & $6,00 \%(3,36-11,3)$ & $7,5 \%(1,7-9,3)$ \\
\hline $\mathrm{N}^{\circ}$ batteriemie polimicrobiche & 170 & 51 \\
\hline$\%$ polimicrobiche/batteriemie significative & $5,61 \%(0-11,6)$ & $5,6 \%(3,2-8,0)$ \\
\hline N. batteriemia nosocomiali & 1614 & n.v. \\
\hline N. batteriemia comunitarie & 1419 & n.v. \\
\hline Batteriemie nosocomiali / I.000 ricoveri & $3,71(1,83-7,35)$ & n.v. \\
\hline Batteriemie comunitarie / I.000 ricoveri & $3,27(1,61-4,40)$ & n.v. \\
\hline
\end{tabular}

Tabella 2. Epidemiologia per reparti

\begin{tabular}{|c|c|c|c|}
\hline & MEDICINE & CHIRURGIE & TERAPIE INTENSIVE \\
\hline $\mathrm{N}^{\circ}$ ricoveri > I giorno & 165.590 & 223.710 & 8.234 \\
\hline $\mathrm{N}^{\circ}$ episodi infettivi & 14.987 & 3.684 & 2.260 \\
\hline Episodi infettivi $\times 1.000$ ricoveri & $90,5(58,7-167)$ & $16,46(10,63-21,9)$ & $274,5(103,4-399,6)$ \\
\hline N.prelievi emocolture per episodio & $2,3(1,77-2,99)$ & $2,01(1,22-2,93)$ & $2,7 \mid(1,48-3,75)$ \\
\hline $\mathrm{N}^{\circ}$ batteriemie significative & 1822 & 631 & 389 \\
\hline$\%$ batteriemie /episodi infettivi & $12,50(9,37-19,6)$ & $I 7, I(10, \mid-25, I)$ & $|7,2|(|2,8-3|, 84)$ \\
\hline $\mathrm{N}^{\circ}$ pseudobatteriemie & 782 & 189251 & 248 \\
\hline \% pseudobatteriemie / episodi infettivi & $5,22(3,22-9,25)$ & $5,13(2,63-8,61)$ & $\mathrm{II}, \mathrm{I}(2,85-\mid 4,56)$ \\
\hline $\mathrm{N}^{\circ}$ batteriemie polimicrobiche & 85 & 55 & 26 \\
\hline$\%$ polimicrobiche/batteriemie significative & $4,66(0-11,73)$ & $8,7(0-15,78)$ & $6,68(0-22,2)$ \\
\hline N. batteriemia nosocomiali & 782 & 437 & 324 \\
\hline N. batteriemia comunitarie & 1040 & 194 & 65 \\
\hline Batteriemie nosocomiali / I.000 ricoveri & $4,72(I, 90-8,5 I)$ & $1,96(0,66-4,13)$ & $39,30(I I, 8-8 I, 84)$ \\
\hline Batteriemie comunitarie / I.000 ricoveri & $6,28(2,64-9,67)$ & $0,86(0,37-1,60)$ & $7,90(2,95-14,4)$ \\
\hline
\end{tabular}


Tabella 3. Eziologia

\begin{tabular}{lcccc}
\hline & $\begin{array}{c}\text { TOTALE } \\
(\mathrm{n} .3 .149 \mathrm{ceppi}) \\
\%\end{array}$ & $\begin{array}{c}\text { NOSOCOMIALI } \\
(\mathrm{n} .1688 \text { ceppi }) \\
\%\end{array}$ & $\begin{array}{c}\text { COMUNITARIE } \\
(\mathrm{n} .146 \text { I ceppi }) \\
\%\end{array}$ & $\begin{array}{c}\text { Rapporto } \\
\text { Noso/Comunitarie } \\
\%\end{array}$ \\
\hline E.coli & 22,5 & 13,0 & 33,3 & 0,45 \\
\hline S.aureus & 17,7 & 21,1 & 13,8 & 1,76 \\
\hline Klebsiella/Enterobacter/Serratia & 10,6 & 14,8 & 5,8 & 2,94 \\
\hline Stafilococchi coagulasi negativi & 9,9 & 14,7 & 4,4 & 3,81 \\
\hline Enterococchi & 6,9 & 8,3 & 5,3 & 1,81 \\
\hline S.pneumoniae & 4,9 & 1,1 & 9,3 & 3,34 \\
\hline Ps.aeruginosa & 5,0 & 6,8 & 2,9 & 7,29 \\
\hline Lieviti & 4,5 & 7,3 & 1,2 & 1,23 \\
\hline Anaerobi & 3,4 & 3,5 & 3,3 & 0,04 \\
\hline Gram negativi non fermentanti & 2,4 & 3,0 & 1,7 & 1,03 \\
\hline Streptococchi alfa emolitici & 3,5 & 2,3 & 4,9 & \\
\hline Proteus spp & 1,8 & 1,7 & 1,9 & 0,8 \\
\hline Salmonella spp. & 1,6 & 0,0 & 3,5 & 0,22 \\
\hline S.agalactiae & 1,1 & 0,9 & 1,4 & \\
\hline Altri batteri & 4,1 & 1,0 & 7,3 & \\
\hline
\end{tabular}

Tabella 4. Eziologia per reparti

\begin{tabular}{lcccccccc}
\hline & Medicine & \multicolumn{2}{c}{ Chirurgie } & \multicolumn{2}{c}{ Terapie intensive } & \multicolumn{2}{c}{ Pediatrie } \\
& n. & $\%$ & n. & $\%$ & n. & $\%$ & n. & $\%$ \\
\hline E.coli & 495 & 26,4 & 149 & 22,2 & 24 & 5,9 & 39 & 20,0 \\
\hline S.aureus & 347 & 18,5 & 113 & 16,8 & 77 & 18,9 & 21 & 10,8 \\
\hline Klebsiella/Enterobacter/Serratia & 128 & 6,8 & 91 & 13,5 & 104 & 25,5 & 12 & 6,2 \\
\hline Stafilococchi coagulasi negativi & 190 & 10,1 & 39 & 5,8 & 75 & 18,4 & 9 & 4,6 \\
\hline Enterococchi & 122 & 6,5 & 59 & 8,8 & 27 & 6,6 & 9 & 4,6 \\
\hline S.pneumoniae & 111 & 5,9 & 6 & 0,9 & 8 & 2,0 & 29 & 14,9 \\
\hline Ps.aeruginosa & 82 & 4,4 & 29 & 4,3 & 43 & 10,5 & 3 & 1,5 \\
\hline Lieviti & 52 & 2,8 & 68 & 10,1 & 12 & 2,9 & 9 & 4,6 \\
\hline Anaerobi & 56 & 3,0 & 41 & 6,1 & 6 & 1,5 & 4 & 2,1 \\
\hline Gram negativi non fermentanti & 37 & 2,0 & 21 & 3,1 & 12 & 2,9 & 6 & 3,1 \\
\hline Streptococchi alfa emolitici & 78 & 4,2 & 21 & 3,1 & 9 & 2,2 & 2 & 1,0 \\
\hline Proteus spp & 32 & 1,7 & 21 & 3,1 & 2 & 0,5 & 2 & 1,0 \\
\hline Salmonella spp. & 30 & 1,6 & 4 & 0,6 & 1 & 0,2 & 16 & 8,2 \\
\hline S.agalactiae & 20 & 1,1 & 1 & 0,1 & 2 & 0,5 & 13 & 6,7 \\
\hline Altri batteri & 94 & 5,0 & 9 & 1,3 & 6 & 1,5 & 21 & 10,8 \\
\hline TOTALE & 1874 & 100 & $\mathbf{6 7 2}$ & 100 & $\mathbf{4 0 8}$ & 100 & 195 & 100 \\
\hline
\end{tabular}

Tabella 5. Eziologia per reparti e nosocomiali/comunitarie

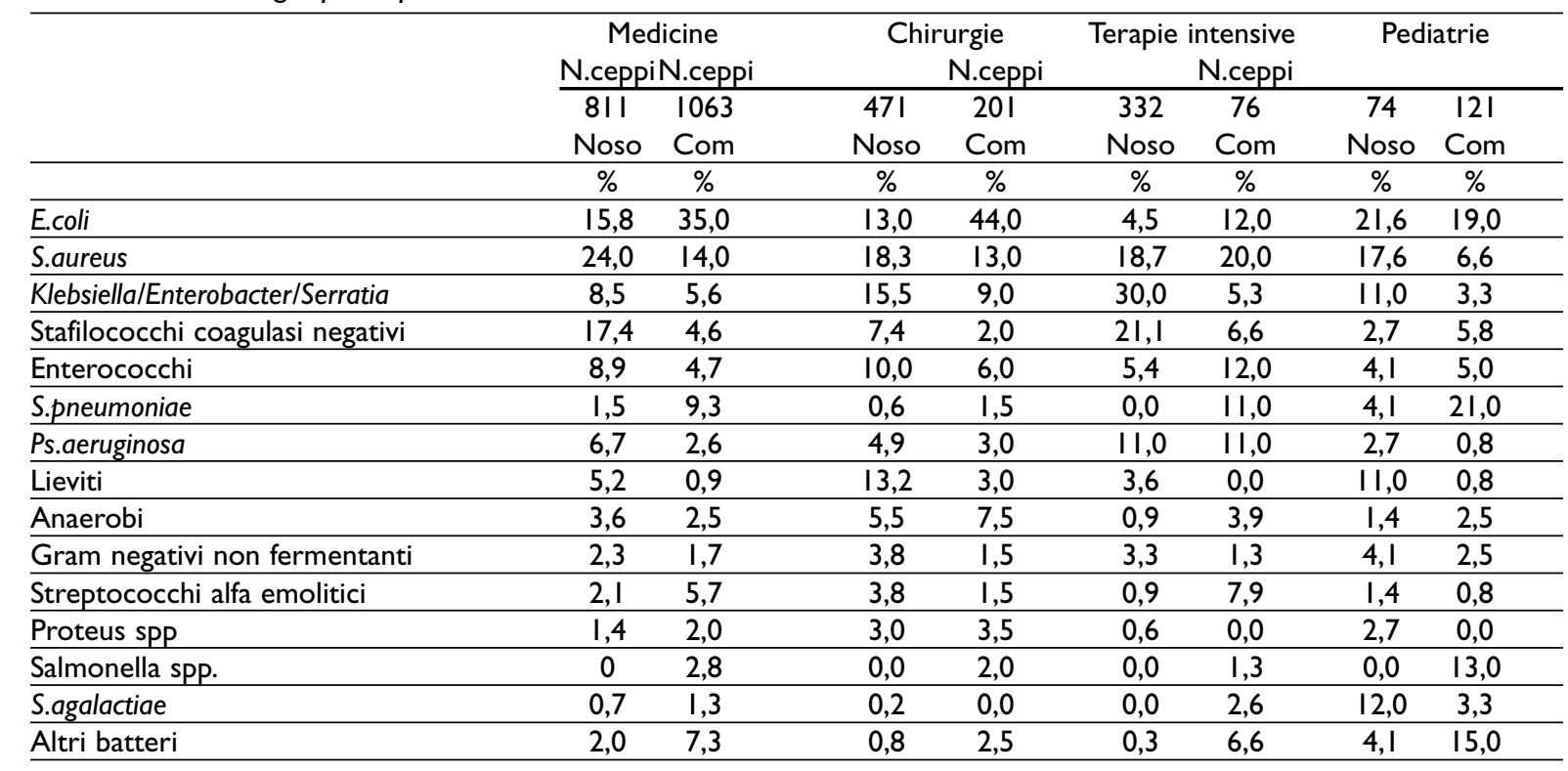




\section{DISCUSSIONE}

Una prima considerazione in merito alla epidemiologia delle batteriemie nei nostri 8 Ospedali è la stabilità dei risultati nei tre anni di osservazione, a conferma della solidità della raccolta dati e della metodologia di calcolo.

Infatti non solo non si sono osservati significativi spostamenti dal 1999 al 2001 ,ma i dati dell'ultimo triennio sono sostanzialmente poco diversi da quelli raccolti nel 1997 in 8 Ospedali Lombardi (di cui 4 partecipanti all'attuale studio), come si vede in tabella 1 .

Importante è sottolineare la dimensione del campione studiato : sono stati osservati 434.466 ricoveri di $>1$ giorno, che rappresentano quasi $1 / 3$ dei ricoveri annui in Lombardia (1.413.129 ricoveri nel 2001) (10) e sono stati documentati 3.033 casi di batteriemia.

Gli Ospedali coinvolti hanno dimensioni mediograndi, con ricoveri $>1$ giorno nel 2001 variabili da 11.600 a 27.600 .

Dalla statistiche della Regione Lombardia si vede che gli Ospedali con $>10.000$ ricoveri annui hanno realizzato nel 2001 un totale di 927.654 ricoveri annui.

Se applicassimo a questo numero di ricoveri i tassi osservati nei nostri 8 Ospedali, ne ricaveremmo queste cifre : 52.273 episodi infettivi indagati, 109.774 prelievi per emocolture, 6475 batteriemie diagnosticate, di cui 3441 nosocomiali. Questo significherebbe un extracosto sanitario , applicando un costo medio di 10.000 euro per caso, calcolabile in 30 milioni di euro annui.

Se applicassimo a questi numeri la mortalità cruda per batteriemia nosocomiale del $27 \%$ osservata in USA nello SCOPE project (Surveillance and Control of Pathogens of Epidemiologic Importance) da Edmond (11) potremmo avere ogni anno in Lombardia 929 morti per batteriemia nosocomiale. Sempre dai dati SCOPE si stima che il $70 \%$ delle batteriemie nosocomiali si osservano in pazienti portatori di cateteri vascolari : una buona parte di queste infezioni potrebbe essere evitata con una adeguata sorveglianza e gestione del catetere vascolare.

Quindi i nostri dati ci danno indicazioni di un fenomeno rilevante per gravità e costi: ma è probabile che la realtà sia ancora peggiore. Solo una raccolta dati con questa metodologia, allargata ad un numero maggiore di ospedali e completata con una analisi per DRG ed esiti potrà dare elementi certi della dimensione del fenomeno, consentirne il monitoraggio negli anni nel singolo Ospedale e nella Regione e dare indicazioni per gli interventi preventivi.

Una seconda considerazione riguarda l'analisi epidemiologica per raggruppamenti di reparto.
È ben evidente dai nostri dati che $\mathrm{i}$ tassi di batteriemia nosocomiale in Terapia Intensiva sono 10 volte superiori a quelli medi dell'Ospedale: i raggruppamenti Medicina e Chirurgia comprendono in realtà reparti molto diversi quanto a rischio infettivo (basti pensare ad esempio ad una Medicina Oncologica rispetto ad una Cardiologia). Allora una raccolta dati su un numero considerevole di Ospedali consentirebbe di analizzare i dati per più raggruppamenti omogenei o per singoli reparti e quindi di stimare il tasso di batteriemie per singole specialità.

Una terza considerazione è che questa raccolta dati ha avuto ricadute importanti all'interno di ogni ospedale, consentendo una analisi attenta della propria realtà ed uno studio del trend nel tempo: infatti localmente ogni microbiologia ha potuto riferire $i$ dati ai clinici, offrendo anche il confronto con altre realtà Lombarde,fornendo indicazioni importanti in termini di eziologia e di terapia empirica.

A proposito dell'eziologia delle nostre batteriemie sono a mio giudizio da evidenziare il ruolo primario dello S.aureus nella responsabilità delle batteriemie nosocomiali, in accordo con molti dati della letteratura, vista la gravità clinica della sepsi da S.aureus: Fowler ( 12 ) ha evidenziato che la complicanza in corso di batteriemia da S.aureus, studiata su oltre 350 casi e definita in base alla presenza di uno di questi esiti (mortalità attribuibile, infezione complicata, stroke embolico, infezione ricorrente durante 12 settimane di follow-up), era presente nel $43 \%$ dei casi.

Altri due aspetti rilevanti dell'eziologia nosocomiale sono il 7,3\% di infezioni da lieviti ed il 3,5 $\%$ di infezioni da anaerobi. In questi casi oltre all'aspetto clinico c'è da considerare l'esigenza di disporre di tecniche diagnostiche ottimali per la ricerca di anaerobi e lieviti: da non trascurare è la precisione diagnostica, che richiede l'utilizzo di adatte procedure di sottocoltura, con più terreni arricchiti e selettivi, visto che questi batteri sono responsabili del $35 \%$ delle batteriemie polimicrobiche ( 8 ), che rappresentano il 5,6\% delle nostre setticemie.

Un ultimo commento in merito alla eziologia riguarda gli Stafilococchi coagulasi negativi, che rappresentano il 9,9\% delle batteriemie ed il 14,7 di quelle nosocomiali: percentuale rilevante ma certamente inferiore al 31,9\% delle batteriemie nosocomiali dello SCOPE project.

Al di là delle differenze tra i nostri ospedali e quelli americani dello studio SCOPE credo che una spiegazione la possa avere il rigore con cui abbiamo escluso dalle batteriemie significative tutti i casi in cui si era avuto un solo prelievo positivo su due o tre oppure si era positivizzato l'uni- 
co prelievo effettuato. Questa esclusione ha determinato ovviamente l'aumento delle pseudobatteriemie o contaminazioni, che nella nostra casistica sono il $6 \%$, un livello inaccettabilmente elevato anche se inferiore al $7,5 \%$ che avevamo osservato nello studio del 1997.

Questo significa che va migliorata la fase di preparazione al prelievo per la emocoltura (disinfezione e dispositivi di prelievo) e va abbandonata l'abitudine, ancora oggi frequente, ad effettuare prelievi solitari per la emocoltura.

A questo proposito, uno studio (13) condotto in USA sulla "Solitary Blood Culture" (SBC) , monitorando questo parametro come indicatore di qualità preanalitica, aveva dimostrato che nel 1996 su 289.572 prelievi per emocoltura condotti su adulti in oltre 900 Ospedali la percentuale di SBC era del $10 \%$.

Nel 2001 una analoga indagine (14) condotta su 333 piccoli Ospedali aveva individuato una mediana per SBC del $12 \%$.

La nostra realtà è probabilmente ben peggiore, come dimostrato in alcuni Ospedali Lombardi che superano ampiamente negli adulti il $40 \%$ di SBC (15).

Infine, per ragioni di spazio, non abbiamo presentato i dati relativi alle resistenze antibiotiche, recentemente pubblicati in un nostro lavoro (9) che aveva evidenziato resistenze in linea con la letteratura.

\section{CONCLUSIONI}

La nostra metodologia di raccolta dati sulla epidemiologia delle batteriemie si è dimostrata solida e consente confronti significativi nel tempo tra Ospedali partecipanti allo studio e nei confronti della letteratura.

È auspicabile che questa raccolta dati possa coinvolgere nei prossimi anni molti altri Ospedali della Lombardia e di altre Regioni ,per rendere ancora più precisi $i$ dati relativi ai tassi di infezione e significativi i confronti.

Vista l'importanza sul piano clinico della batteriemia è auspicabile che i dati della Microbiologia possano essere arricchiti da pochi ma significativi dati clinici ricavabili dai DRG di ricovero per poter stimare anche l'impatto della batteriemia nosocomiale sui costi di degenza ed i suoi esiti e poter misurare gli effetti di eventuali interventi preventivi o terapeutici.

\section{BIBLIOGRAFIA}

1. Wenzel RP, Edmond MB. the impact of Hospitalacquired bloostream infections. Emerg Infect Dis $2001 ; 2$ : 174-177

2. Pittet D. Nosocomial bloostream infections. In : Wenzel RP, Prevention and control of nosocomial infections. $3^{\text {rd }}$ ed. Baltimore : Williams and Wilkins, 1997 : 711-69

3. Pittet D., Tararra D., Wenzel RP. Nosocomial bloodstrema infection in critically ill patients: excess length of stay,extra costs and attributable mortality. JAMA $1994 ; 271: 1598-601$

4. Banerjee S., Emori G., Culver D. et al. Secular trends in Nosocomial primary bloodstream infections in the United States 1980-1989 . Am J Med 1991 ; 91: 86S$89 \mathrm{~S}$

5. Bouza E., Perez-Molina J., Munoz P. Report of ESGNI - 001 and ESGNI - 002 studies. Bloodstream infections in Europe. Clin Microbiol Infect 1999; 5 : 2S1-2S12

6. Privitera G. (in corso di stampa)

7. Lizioli A, Privitera G, Allata E, Banfi EM, Boselli L, Panzeri MC, Perna MC, Porretta AD, Santini MG, Carreri V. Prevalence of nosocomial infections in Italy: result from the Lombardy survey in 2000. J Hosp Infect. 2003; 54(2): 141-8.

8. Viganò EF., Fossati D., Arghittu M., et al Epidemiologia delle batteriemia in otto ospedali della Lombardia . Microbiologia Medica 1999; 14 : 280287

9. Luzzaro F., Viganò EF., Fossati D., et al. Prevalence and drug susceptibility of pathogens causing bloodstream infections in Northern Italy : a two-year study in 16 Hospitals Eur Jclin Microbiol Infect Dis 2002;21:849-855

10. Pubblicazioni della Direzione Generale Sanità, Rapporto sui ricoveri in Lombardia 2001, Tabella 2.2.2

11. Edmond MB,Fallace SE, McClish DK,Pfaller MA, Jones RN, Wenzel RP Nosocomial Bloodstream Infections in United States Hospitals: a three-year analyis CID $1999 ; 29: 239-44$

12. Fowler VG Jr, Olsen MK, Corey GR, Woods CW, Cabell $\mathrm{CH}$, Reller LB, Cheng AC, Dudley T, Oddone EZ Clinical identifiers of complicated Staphylococcus aureus bacteremia Arch Intern Med 2003 ; 163 : 2066 - 72

13. Schifman RB, Bachner RP. Hovanitz PJ - Blood culture quality improvement: a College of American Pathologists Q-Probes study involving 909 institutions and 289572 blood culture sets. Arch Pathol Lab Med. 1996; 120 : 999-1002.

14. Novis DA, Dale JC, Schifman RB, Ruby SG, Walsh MK - Solitary blood cultures: a College of American Pathologists Q-probes study of 132,778 blood culture sets in 333 small hospitals. Arch Pathol Lab Med. 2001; $125: 1290-4$.

15. Viganò EF. Metodologie (convenzionali,automatizzate,molecolari) per la diagnosi di batteriemia. Relazione al Congresso Nazionale AMCLI Rimini 2002.

Egidio Franco Viganò

U.O.Microbiologia - Via Candiani 2 20025 Legnano (MI)

Tel 0331- 449319/364 - Fax 0331-449578

E-mail: egidio.vigano@ao-legnano.it 